\title{
Solving DLP with Auxiliary Input over an Elliptic Curve Used in TinyTate Library
}

\author{
Yumi Sakemi ${ }^{1}$, Tetsuya Izu ${ }^{2}$, Masahiko Takenaka², \\ and Masaya Yasuda ${ }^{2}$ \\ 1 Okayama University \\ 3-1-1, Tsushima-naka, Kita-ku, Okayama, 700-8530, Japan \\ 2 FUJITSU LABORATORIES Ltd., \\ 4-1-1, Kamikodanaka, Nakahara-ku, Kawasaki, 211-8588, Japan \\ $\{$ izu, takenaka, myasuda\}@labs.fujitsu.com
}

\begin{abstract}
The discrete logarithm problem with auxiliary input (DLPwAI) is a problem to find $\alpha$ from $G, \alpha G, \alpha^{d} G$ in an additive cyclic group generated by $G$ of prime order $r$ and a positive integer $d$ dividing $r-1$. The infeasibility of DLPwAI assures the security of some cryptographic schemes. In 2006, Cheon proposed a novel algorithm for solving DLPwAI. This paper shows our experimental results of Cheon's algorithm by implementing it with some speeding-up techniques. In fact, we succeeded to solve DLPwAI in a group with 128-bit order in 45 hours with a single PC on an elliptic curve defined over a prime finite field with 256-bit elements which is used in the TinyTate library.
\end{abstract}

\section{Introduction}

Let $\mathbb{G}$ be an additive cyclic group generated by $G$ of prime order $r$. The discrete logarithm problem with auxiliary input (DLPwAI) is a problem to find $\alpha$ from $G, \alpha G, \alpha^{d} G \in \mathbb{G}$ and a positive integer $d$ dividing $r-1$. The infeasibility of DLPwAI assures the security of some cryptographic schemes including BonehBoyen's ID-based encryption scheme 2] and Boneh-Gentry-Waters' broadcast encryption scheme [5]. In 2006, Cheon proposed a novel algorithm for solving DLPwAI 78 . The time complexity of Cheon's algorithm (with KKM improvement) is $O(\sqrt{(r-1) / d}+\sqrt{d})$, and especially when $d \approx \sqrt{r}$, the complexity becomes $O(\sqrt[4]{r})$, which is much efficient than that for solving DLP in general groups (which requires $O(\sqrt{r})$ ).

This paper implements Cheon's algorithm combined with the baby-step giantstep algorithm [16] as a sub-algorithm and some speeding-up techniques. Then, this paper reports experimental results of our implementation. In fact, we have successfully solved a DLPwAI in 45 hours with a single PC in a group with 128-bit order defined on an elliptic curve over a prime finite field with 256bit elements, which is used in the Tiny Tate library [14 for implementing pairing cryptosystem in the embedded devices (see also Table1, Note that Jao-Yoshida's 
Table 1. Required time for solving DLPwAI

\begin{tabular}{l|r|r}
\hline & Size of $r$ & Required Time \\
\hline Jao, Yoshida [1] & $60 \mathrm{bit}$ & 3 hours \\
\hline Izu et al. [10] & $83 \mathrm{bit}$ & 14 hours \\
\hline This paper & $\mathbf{1 2 8} \mathbf{b i t}$ & $\mathbf{4 5}$ hours \\
\hline
\end{tabular}

result was not dedicated to an efficient implementation. Also note that Izu et al.'s result was implemented over a finite field with characteristics 3). Here, solving DLP on the elliptic curve is regarded to be infeasible (since the order is 128bit). As a feedback of our experimental results, it is better to avoid such elliptic curve when some cryptographic schemes are implemented. We also estimated the required time and memory for solving DLPwAI with larger $r$. According to our estimations, it would be difficult to solve DLPwAI with larger $r$ by the same approach, namely, Cheon's algorithm combined with the baby-step giant-step algorithm.

\section{Preliminaries}

This section introduces the discrete logarithm problem with auxiliary input (DLPwAI) and Cheon's algorithm for solving DLPwAI 7/8. Implications of DLPwAI and Cheon's algorithm on cryptographic schemes are also explained.

\subsection{Discrete Logarithm Problem with Auxiliary Input (DLPwAI)}

Let $\mathbb{G}=\langle G\rangle$ be an additive group generated by $G$ of prime order $r$. The discrete logarithm problem (DLP) in $\mathbb{G}$ is to find $\alpha \in \mathbb{Z} / r \mathbb{Z}$ on input $G, \alpha G \in \mathbb{G}$. In the general setting, the most efficient algorithms for solving DLP require $O(\sqrt{r})$ in time. In fact, Shanks' baby-step giant-step (BSGS) algorithm [16] requires $O(\sqrt{r})$ group operations in time and $O(\sqrt{r})$ group elements in space. On the other hand, Pollard's $\lambda$-algorithm also requires $O(\sqrt{r})$ in time, but much smaller elements in space.

In 2006, Cheon defined the discrete logarithm problem with auxiliary input (DLPwAI) as a variant of DLP [7/8, where DLPwAI is a problem to find $\alpha$ on input $G, \alpha G, \alpha^{d} G$ and an integer $d$ dividing $r-11$. At the same time, Cheon proposed a novel algorithm for solving DLPwAI 7/8. Cheon's algorithm (together with Kozaki-Kutsuma-Matsuo's improvement [12]) requires $O(\sqrt{(r-1) / d}+\sqrt{d})$ group operations in time. Especially, when $d \approx \sqrt{r}$, it only requires $O(\sqrt[4]{r})$ operations, which is much smaller than that required in the baby-step giant-step algorithm or in the $\lambda$-algorithm for solving DLP.

${ }^{1}$ There are many possible variations of DLPwAI. However, this paper only deals with this type. 


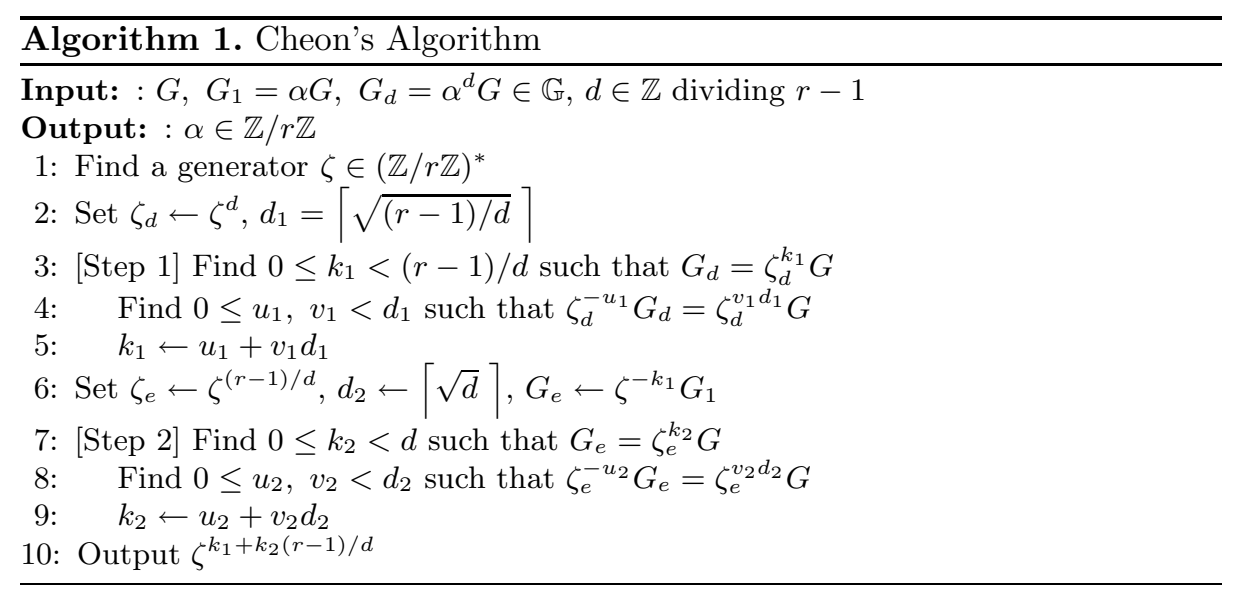

\subsection{Cheon's Algorithm}

A goal of Cheon's algorithm is to find an integer $k \in \mathbb{Z} / r \mathbb{Z}$ such that $\alpha=\zeta^{k}$ for a generator of the multiplicative group $\zeta \in(\mathbb{Z} / r \mathbb{Z})^{*}$ (Note that finding the generator $\zeta$ is easy). Here, such $k$ is uniquely determined. To do so, Cheon's algorithm searches two integers $k_{1}, k_{2}$ such that $k=k_{1}+k_{2}(r-1) / d$ satisfying $0 \leq k_{1}<(r-1) / d, 0 \leq k_{2}<d$ in the following two steps (see Algorithm 1).

Step 1 searches an integer $k_{1}$ such that $\alpha^{d}=\zeta_{d}^{k_{1}}$ for $\zeta_{d}=\zeta^{d}$, or equivalently, searches two integers $u_{1}, v_{1}$ such that

$$
\alpha^{d} \zeta_{d}^{-u_{1}}=\zeta_{d}^{v_{1} d_{1}}
$$

satisfying $0 \leq u_{1}, v_{1}<\sqrt{(r-1) / d}$. Here, such $u_{1}, v_{1}$ are uniquely determined. In practice, Step 1 searches $u_{1}, v_{1}$ such that $\zeta_{d}^{-u_{1}} G_{d}=\zeta_{d}^{v_{1} d_{1}} G$.

Then, Step 2 searches an integer $k_{2}$ such that $\alpha=\zeta^{k_{1}} \zeta_{e}^{k_{2}}$ for $\zeta_{e}=\zeta^{(r-1) / d}$ in the similar way, or equivalently, searches integers $u_{2}, v_{2}$ such that

$$
\alpha \zeta^{-k_{1}} \zeta_{e}^{-u_{2}}=\zeta_{e}^{v_{2} d_{2}}
$$

satisfying $0 \leq u_{2}, v_{2}<\sqrt{d}$ (where $G_{e}=\zeta^{-k_{1}} G$ ). Here, such $u_{2}, v_{2}$ are uniquely determined. In practice, Step 2 searches $u_{2}, v_{2}$ such that $\zeta_{e}^{-u_{2}} G_{1}=\zeta_{e}^{v_{2} d_{2}} G$.

In Cheon's algorithm, searching $u_{1}, v_{1}$ in Step 1 and searching $u_{2}, v_{2}$ in Step 2 require another sub-algorithm. Since these problems are very similar to DLP in the general setting, the baby-step giant-step algorithm or the $\lambda$-algorithm can be used as a subroutine. Since this paper is interested in Cheon's algorithm combined with the baby-step giant-step algorithm only, the next section briefly describes its outline.

Baby-step Giant-step Algorithm. The baby-step giant-step (BSGS) algorithm was introduced by Shanks in 1971 for solving DLP [16]. Instead of finding $\alpha$ directly, on input $G$ and $G_{1}=\alpha G$, BSGS searches two integers $i, j$ such 
that $\alpha=i+j m$ and $0 \leq i, j<m=\lceil\sqrt{r}$. Here, such $i, j$ are uniquely determined. Since $\alpha G=(i+j m) G=i G+j G^{\prime}$ for $G^{\prime}=m G$, we have a relation $G_{1}-i G=j G^{\prime}$.

BSGS consists of two steps: in the 1st step (the baby-step), we compute

$$
G_{1}, G_{1}-G, G_{1}-2 G, \ldots, G_{1}-(m-1) G
$$

successively and store them in a database. In the 2nd step (the giant-step), we compute

$$
G^{\prime}, 2 G^{\prime}, \ldots,(m-1) G^{\prime}
$$

successively and store them in another database. Then, we search a collision $G_{1}-i G=j G^{\prime}$ among these databases and thus a solution $\alpha=i+j m$ is obtained. Since $O(m)$ group operations and $O(m)$ group elements are required in both steps, the time and space complexity of BSGS are $O(\sqrt{r})$ group operations and $O(\sqrt{r})$ group elements, respectively.

When BSGS algorithm is used in Step 1 of Cheon's algorithm, we establish two databases

$$
\zeta_{d}^{0} G_{d}, \zeta_{d}^{-1} G_{d}, \zeta_{d}^{-2} G_{d}, \ldots, \zeta_{d}^{-d_{1}} G_{d}
$$

and

$$
\zeta_{d}^{0} G, \zeta_{d}^{d_{1}} G, \zeta_{d}^{2 d_{1}}, \ldots, \zeta_{d}^{d_{1}^{2}} G
$$

and searches a collision satisfying $\zeta_{d}^{-u_{1}} G_{d}=\zeta_{d}^{v_{1} d_{1}} G$ among these databases. Thus, Step 1 in Cheon's algorithm combined with BSGS algorithm requires $2 d_{1}=2 \sqrt{(r-1) / d}$ elements in space. Similarly, Step 2 requires $2 d_{2}$ elements in space.

KKM Improvement. Cheon's algorithm requires the number of scalar multiplications for fixed elements $G, G_{1}$ and $G_{d}$. In 2007, Kozaki, Kutsuma and Matsuo introduced a precomputation table for such multiplications and reduced the time complexity of Cheon's algorithm from $O(\log r(\sqrt{(r-1) / d}+\sqrt{d}))$ to $O(\sqrt{(r-1) / d}+\sqrt{d})$.

Let us describe KKM improvement for a scalar multiplication $\gamma P(\gamma \in \mathbb{Z} / r \mathbb{Z}$, $P \in \mathbb{G}$ ) in the followings. For a fixed integer $c$ (which will be optimized later) and $n=\lceil\sqrt[c]{r}\rceil$, obtain the $n$-array expansion of the scalar $\gamma=\sum_{i=0}^{c-1} \gamma_{i} n^{i}(0 \leq$ $\left.\gamma_{i}<n\right)$. For all $0 \leq i<c$ and $0 \leq j<n$, compute $S(i, j)=j n^{i} P$ and store them in a table in advance to the scalar multiplication. Then, the scalar multiplication $\gamma P$ is computed by the following way:

$$
\begin{aligned}
\gamma P & =\gamma_{0} P+\gamma_{1} n P+\cdots+\gamma_{c-1} n^{c-1} P \\
& =S\left(0, \gamma_{0}\right)+S\left(1, \gamma_{1}\right)+\cdots+S\left(c-1, \gamma_{c-1}\right)
\end{aligned}
$$

Since the precomputation table can be computed by at most $c$ scalar multiplications and $c n$ additions, KKM improvement reduces the time complexity of Cheon's algorithm by a factor of $\log r$. 
Complexity of Cheon's Algorithm. As a summary of this section, when Cheon's algorithm is combined with the baby-step giant-step algorithm and KKM improvement, the time complexity $T$ and the space complexity $S$ are evaluated by the followings:

$$
\begin{aligned}
& T=O(\sqrt{(r-1) / d}+\sqrt{d}) \quad \text { (group operations), } \\
& S=O(\max (\sqrt{(r-1) / d}, \sqrt{d})) \quad(\text { group elements). }
\end{aligned}
$$

\subsection{DLPwAI in Cryptographic Schemes}

In recently proposed cryptographic schemes, new mathematical problems have been proposed and the infeasibility of such problems assure the security of the schemes. For example, $\ell$-BDHE problem was introduced in Boneh-GentryWaters' broadcast encryption system [5]. Here, $\ell$-BDHE problem is a problem to find $e(G, \hat{G})^{\alpha^{\ell+1}}$ for a given bilinear map $e: \mathbb{G} \times \hat{\mathbb{G}} \rightarrow \mathbb{G}_{T}$ on input $G, \alpha G, \ldots, \alpha^{\ell} G, \alpha^{\ell+2} G, \ldots, \alpha^{2 \ell} G \in \mathbb{G}$ and $\hat{G} \in \hat{\mathbb{G}}$, where $\mathbb{G}=\langle G\rangle, \hat{\mathbb{G}}=\langle\hat{G}\rangle$, and $\mathbb{G}_{T}$ is a multiplicative group with order $r$. When $\ell>d$, Cheon's algorithm can be applied to the scheme: by finding $\alpha$ as DLPwAI, an answer of $\ell$-BDHE problem is obtained. Thus, Cheon's algorithm is an attacking algorithm for such cryptographic schemes. Note that there are many other recently proposed problems to which Cheon's algorithm can be allied such as $\ell$-SDH problem [3], $\ell$-sSDH problem [4], $\ell$-BDHI problem [2].

\section{Implementation}

This section describes our strategy for implementing Cheon's algorithm. We adopted the baby-step giant-step (BSGS) algorithm as a subroutine, and KKM improvement for the speeding-up.

\subsection{BSGS Algorithm}

Databases. In step 1 of Cheon's algorithm, when BSGS algorithm is used, two databases

$$
\mathrm{DB}_{1, \mathrm{~B}}=\left\{\zeta_{d}^{0} G_{d}, \zeta_{d}^{-1} G_{d}, \ldots, \zeta_{d}^{-i} G_{d}, \ldots, \zeta_{d}^{-d_{1}} G_{d}\right\}
$$

and

$$
\mathrm{DB}_{1, \mathrm{G}}=\left\{\zeta_{d}^{0} G, \zeta_{d}^{d_{1}} G, \ldots, \zeta_{d}^{j d_{1}} G, \ldots, \zeta_{d}^{d_{1}^{2}} G\right\}
$$

should be established. In our implementation, an element $\zeta_{d}^{-i} G_{d}$ in the database $\mathrm{DB}_{1, \mathrm{~B}}$ is expressed by the following 12-byte representation

$$
\underbrace{i}_{\text {4-byte }} \| \underbrace{\operatorname{LSB}_{64}\left(\operatorname{MD} 5\left(x\left(\zeta_{d}^{-i} G_{d}\right) \| y\left(\zeta_{d}^{-i} G_{d}\right)\right)\right)}_{\text {8-byte }}
$$


where $i$ is assumed to be 4 -byte, $\operatorname{LSB}_{64}(\cdot)$ represents the least 64-bit (8-byte) of the data, MD5 is the hash function, and $x(G), y(G)$ represent $x$ - and $y$ coordinate values of a point $G$ on an elliptic curve. In the following, $i$ is identified as the index part and the rest is as the data part. Similarly, in the database $\mathrm{DB}_{1, \mathrm{G}}$, an element $\zeta_{d}^{j d_{1}} G$ is expressed by the following 12 -byte representation

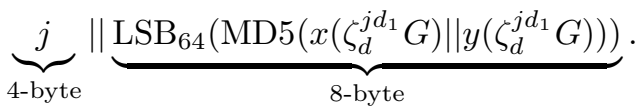

Thus, the databases $\mathrm{DB}_{1, \mathrm{~B}}$ and $\mathrm{DB}_{1, \mathrm{G}}$ requires $12 d_{1}$ bytes, respectively.

Database Search. Then, in step 1 of Cheon's algorithm, it is needed to find two integers $u_{1}, v_{1}$ satisfying $\zeta_{d}^{-u_{1}} G_{d}=\zeta_{d}^{v_{1} d_{1}} G$ among the databases $\mathrm{DB}_{1, \mathrm{~B}}$ and $\mathrm{DB}_{1, \mathrm{G}}$. Since a straight-forward search (namely, compare all $\zeta_{d}^{-i} G_{d}$ in $\mathrm{DB}_{1, \mathrm{~B}}$ and $\zeta_{d}^{j d_{1}} G$ in $\left.\mathrm{DB}_{1, \mathrm{G}}\right)$ is inefficient, we used the following bucket-sort like algorithm [1].

1. Divide each database into 64 sub-databases depending on the most significant 6 -bit of the index part. When the most significant 6 -bit of data $\zeta_{d}^{-i} G_{d}$ in $\mathrm{DB}_{1, \mathrm{~B}}$ is $\ell(0 \leq \ell \leq 63)$, it is stored in the sub-database $\mathrm{DB}_{1, \mathrm{~B}}^{(\ell)}$. Similarly, when the most significant 6 -bit of data $\zeta_{d}^{j d_{1}} G$ in $\mathrm{DB}_{1, \mathrm{G}}$ is $\ell(0 \leq \ell \leq 63)$, it is stored in the sub-database $\mathrm{DB}_{1, \mathrm{G}}^{(\ell)}$.

2. Sort all sub-database $\mathrm{DB}_{1, \mathrm{G}}^{(\ell)}(\ell=0, \ldots, 63)$. by the comb-sort algorithm 6 , which sorts $N$ elements in $O(\log N)$.

3. For each $\ell$, search a collision among $\mathrm{DB}_{1, \mathrm{~B}}^{(\ell)}$ and $\mathrm{DB}_{1, \mathrm{G}}^{(\ell)}$. To do so, pick up an element $\zeta_{d}^{j d_{1}} G$ from the sub-database $\mathrm{DB}_{1, \mathrm{~B}}^{(\ell)}$ and check whether the same element is in $\mathrm{DB}_{1, \mathrm{G}}^{(\ell)}$ by the binary search algorithm.

If a collision is found in step 3 for a certain $\ell$, then, their indexes are what we required: set $u_{1} \leftarrow i$ and $v_{1} \leftarrow j$.

\subsection{KKM Improvement}

In our implementation, KKM improvement is also used for speeding-up Cheon's algorithm. Since our target group $\mathbb{G}$ is on an elliptic curve defined over a prime finite field with a mediate size, the affine coordinate system is used rather than the projective coordinate system. In the affine coordinate, every elliptic curve addition requires an inversion computation in the finite field. In order to avoid heavy operations, we used the Montgomery trick [13], which converts $N$ inversion computations into 1 inversion and $3(N-1)$ multiplication computations. When the Montgomery trick is used in KKM improvement, only $O\left(\log _{2} c\right)$ inversions are required. 


\section{Experimental Results}

This section describes our experimental results of Cheon's algorithm for an elliptic curve used in the TinyTate library [14]. We successfully solved DLPwAI by our implementation in a group $\mathbb{G}$ with 128 -bit order. Strongly note that DLP has been believed to be secure in the same group.

\subsection{Parameters}

We used an addition cyclic group $\mathbb{G}=\langle G\rangle$ with order $r$ on an elliptic curve $y^{2}=x^{3}+x$ defined over a prime finite field $\mathbb{F}_{p}$ used in the TinyTate library [14] which was developed by Oliveria et al. for implementing the pairing-based cryptosystem in the embedded devices. Concrete values of these parameters are summarized in the following:

$$
\begin{aligned}
& p=0 \times 5387 \mathrm{a} 1 \mathrm{~b} 6 \text { 93d85f28 8f131dd5 e7f9305c f4436019 a00f3181 } \\
& 168 \mathrm{~d} 7 \mathrm{~b} 208934 \mathrm{~d} 073 \text { (256-bit) } \\
& =37781606889598235856745576472658394721481625071533 \\
& 302983957476142038207746163 \\
& \# E=0 x 5387 \mathrm{a} 1 \mathrm{~b} 6 \text { 93d85f28 8f131dd5 e7f9305c f4436019 a00f3181 } \\
& \text { 168d7b20 8934d074 (256-bit) } \\
& =37781606889598235856745576472658394721481625071533 \\
& 302983957476142038207746164 \\
& =2^{2} \cdot 3^{2} \cdot 1227703 \cdot 5024295160706223327986689772851 \cdot r \\
& r=0 \times 80000040000000000000000000000001 \text { (128-bit) } \\
& =170141188531071632644604909702696927233 \\
& r-1=0 \times 80000040000000000000000000000000 \text { (128-bit) } \\
& =2^{102} \cdot 3 \cdot 11 \cdot 251 \cdot 4051
\end{aligned}
$$

where $\# E$ denotes the number of points in $E\left(\mathbb{F}_{p}\right)$. In our implementation of Cheon's algorithm, we used the following parameters:

$$
\begin{aligned}
d & =12682136550675316736 \quad \text { (64-bit) } \\
& =2^{60} \cdot 11 \\
\zeta & =5 \\
\zeta_{d} & =\zeta^{d}=124313318310214169447902414199634645036 \\
d_{1} & =\lceil\sqrt{(r-1) / d}\rceil=3662760472 \quad \text { (32-bit) } \\
d_{2} & =\lceil\sqrt{d}\rceil=3561198752 \quad \text { (32-bit) }
\end{aligned}
$$

Here, $d$ is chosen to minimize the time complexity of Cheon's algorithm, and it is estimated that our implementation requires about $O\left(2^{32.75}\right)$ group operations 
Table 2. Computational Environment

\begin{tabular}{c|c}
\hline CPU & Intel(R) Core $^{\mathrm{TM}}$ i7 $2.93 \mathrm{GHz}$ \\
OS & Ubuntu 10.04 \\
Compiler & gcc 4.2 .2 \\
Library & GNU MP $4.1 .2[9$ \\
\hline
\end{tabular}

and elements for solving DLPwAI. The generator $\zeta$ is chosen as the minimum generator of the multiplicative group $(\mathbb{Z} / r \mathbb{Z})^{*}$.

A base point $G$ is randomly chosen from points in $E\left(\mathbb{F}_{p}\right)$ with order $r$. Then, coordinate values of $G, G_{1}=\alpha G, G_{d}=\alpha^{d} G$ for our solution $\alpha=3$ are as follows:

$$
\begin{aligned}
x(G)= & 21200288773256318148725438778421364777055392159948 \\
& 43243899490272291266930386 \\
y(G)= & 26761623705989368931204018789692655222931214614323 \\
& 914063578840689729495767328 \\
x\left(G_{1}\right)= & 14065656213797322149877452698705463657001853001649 \\
& 73389265776415100308801614 \\
y\left(G_{1}\right)= & 38683308574106521926078235874461216295912909889285 \\
& 50616717683614580548353865 \\
x\left(G_{d}\right)= & 32496897821175066681382870355623859749401559994074 \\
& 955520148762053651605880230 \\
y\left(G_{d}\right)= & 20178499008260892062075798558988490926923717554232 \\
& 085908274534745971737681072
\end{aligned}
$$

\subsection{Results}

In the experiment, our implementation of Cheon's algorithm successfully found the solution $\alpha=3$ in about 45 hours and 246 GByte by using a single PC (other environmental information are summarized in Table 2).

KKM Improvement. In our implementation, the Montgomery trick is used for KKM improvement part. By experimental optimizations, we used parameters $n=2^{16}$ and $c=8$. The precomputation requires about 4 seconds, and each scalar multiplication requires about $27 \mu$ seconds. About $1.03 \mathrm{mseconds}$ is required for a multiplication without KKM improvement, about 38 times speedup was established. Also, about $54 \mu$ seconds is required with KKM improvement but without the Montgomery trick, about 2 times speed-up was established.

Step 1. Two databases $\mathrm{DB}_{1, \mathrm{~B}}$ and $\mathrm{DB}_{1, \mathrm{G}}$ are generated in about 14.5 hours and 82 GByte memory with 4 parallel computations. Then, 1 hour is required 
to divide these databases into 64 sub-databases $\mathrm{DB}_{1, \mathrm{~B}}^{(\ell)}$ and $\mathrm{DB}_{1, \mathrm{G}}^{(\ell)}(0 \leq \ell \leq 63)$. Since the parent databases $\mathrm{DB}_{1, \mathrm{~B}}$ and $\mathrm{DB}_{1, \mathrm{G}}$, and their cache are stored in this divisions, $82 \times 3=246$ GByte are required.

Sorting a sub-database $\mathrm{DB}_{1, \mathrm{~B}}^{(\ell)}$ required about 8 minutes, and searching a collision among $\mathrm{DB}_{1, \mathrm{~B}}^{(\ell)}$ and $\mathrm{DB}_{1, \mathrm{G}}^{(\ell)}$ required about 2 minutes. In our experiment, we found a collision when $\ell=39$ and obtained $u_{1}=2170110422$ and $v_{1}=846301393$. Thus, we found a partial solution

$$
\begin{aligned}
k_{1} & =u_{1}+v_{1} d_{1} \\
& =3099799291849047918
\end{aligned}
$$

in about 22.1 hours.

Step 2. Similarly to Step 1, the database generation required about 14.2 hours and 80 GByte. Dividing databases required about 1 hour and $80 \times 3=240$ GByte. Sorting a sub-database required about 7 minutes and searching a collision among sub-databases required about 2 minutes. We found a collision when $\ell=52$ and obtained $u_{2}=1609744154$ and $v_{2}=718704617$. Thus, we found a partial solution

$$
\begin{aligned}
k_{2} & =u_{2}+v_{2} d_{2} \\
& =2559449986726782138
\end{aligned}
$$

in about 23.2 hours.

Consequently, we successfully obtained

$$
\begin{aligned}
k & =k_{1}+k_{2}(r-1) / d \\
& =34337105659404196008394232931084369774
\end{aligned}
$$

and the solution

$$
\alpha=\zeta^{k} \bmod r=3
$$

in about 45.3 hours and 246 GByte memory.

\subsection{Estimations}

Based on our experimental results described in the previous section, we estimate required time (in the worst case) and memory for solving DLPwAI with larger $r$. Here, we do not consider the parallel processing. We assume that the parameter $d$ can be chosen as large as $\sqrt{r}$, namely, required time in Step 1 and Step 2 are almost same.

Let $T_{\mathrm{C}}$ be the required time for generating databases. Since a scalar multiplication is computed in $27 \mu$ seconds, $T_{\mathrm{C}}$ can be evaluated by

$$
\left.T_{\mathrm{C}}=2 \times 27 \times 10^{-6} \times 2 \sqrt[4]{r} \text { [Seconds }\right]
$$


Table 3. Cost Estimations

\begin{tabular}{c|c|c|c|c}
\hline$r$ & $T_{\mathrm{C}}$ & $T_{\mathrm{S}}$ & $T=T_{\mathrm{C}}+T_{\mathrm{S}}$ & $S_{\mathrm{C}}$ \\
\hline 128 bit & 6 Days & 17 Hours & 7 Days & 288 GByte \\
132 bit & 11 Days & 35 Hours & 13 Days & 576 GByte \\
136 bit & 22 Days & 74 Hours & 25 Days & 1152 GByte \\
140 bit & 45 Days & 6 Days & 51 Days & 2304 GByte \\
\hline
\end{tabular}

Table 4. Estimated Time for 128-bit $r$

\begin{tabular}{l|r}
\hline & Estimated Time \\
\hline Jao, Yoshida [1] & 16384 Days \\
\hline Izu et al. [10] & 1195 Days \\
\hline This paper & 7 Days \\
\hline
\end{tabular}

Then, let us evaluate the required time $T_{\mathrm{S}}$ for the database search. Since the sorting are dominant procedures, we neglect time for other parts. In our implementation, the comb-sort algorithm requires $O(\log N)$ for sorting $N$ elements, and a sort in a sub-database requires 8 minutes for 128-bit $r, T_{\mathrm{S}}$ can be evaluated by

$$
\left.T_{\mathrm{S}}=2 \times 64 \times(8 \times 60) \times \frac{(\sqrt[4]{r}) \log (\sqrt[4]{r} / 64)}{\left(2^{32}\right) \log \left(2^{26}\right)} \quad \text { SSeconds }\right] .
$$

On the other hand, the required memory $S_{\mathrm{C}}$ can be evaluated by

$$
S_{\mathrm{C}}=12 \times \sqrt[4]{r} \times 3 \times 2[\text { Bytes }] .
$$

By using these evaluations, estimated required time and memory for various sizes of $r$ are summarized in Table 3

According to Table 3, solving DLPwAI seems to be feasible even if $r$ is 140bit since the required time is about 50 days. However, the required memory is beyond 2 TByte in this case. In the computational environment we used, and in most environments, dealing with such huge memory is too difficult to proceed. Thus, it is concluded that solving DLPwAI with 140-bit $r$ is infeasible by Cheon's algorithm combined with BSGS algorithm. In order to solve such larger problems, Cheon's algorithm combined with the $\lambda$-algorithm or the kangaroo algorithm would be employed.

Next, let us compare our results to the previous experiments (summarized in Table 1) when $r$ is 128-bit by the extrapolation. According to the time complexity of Cheon's algorithm, Jao and Yoshida's implementation 2 would require $3 \times$ $\sqrt{2^{64} / 2^{30}}=393216$ hours (16384 days) and Izu et al.'s implementation would require $14 \times \sqrt{2^{64} / 2^{42}}=28672$ hours (1195 days) (Table 4). Even if the parallel computation is applied, solving DLPwAI is infeasible with 128-bit $r$ by these implementations.

\footnotetext{
${ }^{2}$ Note that Jao-Yoshida's result was not dedicated to an efficient implementation.
} 


\section{Concluding Remarks}

This paper succeeded to solve a discrete logarithm problem with auxiliary input (DLPwAI) in 45 hours with a single PC in a group with 128-bit order defined on an elliptic curve over a prime finite field with 256-bit elements, which are used in TinyTate library for implementing pairing cryptosystem in the embedded devices. If cryptographic schemes based on problems such as $\ell$-BDE problem, $\ell$-SDH problem, $\ell$-sSDH problem or $\ell$-BDHI problem are implemented, TinyTate library should avoid using such weak parameters. However, there are pairingbased cryptographic schemes which are not effected by Cheon's algorithm such as Boneh-Franklin's ID-based encryption scheme.

\section{Acknowledgements}

The authors would like to thank Yoshitaka Morikawa and Yasuyuki Nogami in Okayama University for their supports on this research.

\section{References}

1. Aoki, K., Ueda, H.: Sieving Using Bucket Sort. In: Lee, P.J. (ed.) ASIACRYPT 2004. LNCS, vol. 3329, pp. 92-102. Springer, Heidelberg (2004)

2. Boneh, D., Boyen, X.: Efficient Selective-ID Secure Identity-Based Encryption Without Random Oracles. In: Cachin, C., Camenisch, J.L. (eds.) EUROCRYPT 2004. LNCS, vol. 3027, pp. 223-238. Springer, Heidelberg (2004)

3. Boneh, D., Boyen, X.: Short Signatures Without Random Oracles. In: Cachin, C., Camenisch, J.L. (eds.) EUROCRYPT 2004. LNCS, vol. 3027, pp. 56-73. Springer, Heidelberg (2004)

4. Boneh, D., Boyen, X., Goh, E.-J.: Hierarchical Identity Based Encryption with Constant Size Ciphertext. In: Cramer, R. (ed.) EUROCRYPT 2005. LNCS, vol. 3494, pp. 440-456. Springer, Heidelberg (2005)

5. Boneh, D., Gentry, C., Waters, B.: Collusion Resistant Broadcast Encryption with Short Ciphertexts and Private Keys. In: Shoup, V. (ed.) CRYPTO 2005. LNCS, vol. 3621, pp. 258-275. Springer, Heidelberg (2005)

6. Box, R., et al.: A Fast Easy Sort. Computer Journal of Byte Magazine 16(4), 315-320 (1991)

7. Cheon, J.H.: Security Analysis of the Strong Diffie-Hellman Problem. In: Vaudenay, S. (ed.) EUROCRYPT 2006. LNCS, vol. 4004, pp. 1-11. Springer, Heidelberg (2006)

8. Cheon, J.H.: Discrete Logarithm Problems with Auxiliary Inputs. Journal of Cryptology 23(3), 457-476 (2010)

9. GNU MP, http://gmplib.org/

10. Izu, T., Takenaka, M., Yasuda, M.: Experimental Results on Cheon's Algorithm. In: WAIS 2010 in the Proceedings of ARES 2010, pp. 625-630. IEEE Computer Science, Los Alamitos (2010)

11. Jao, D., Yoshida, K.: Boneh-Boyen Signatures and the Strong Diffie-Hellman Problem. In: Shacham, H., Waters, B. (eds.) Pairing 2009. LNCS, vol. 5671, pp. 1-16. Springer, Heidelberg (2009) 
12. Kozaki, S., Kutsuma, T., Matsuo, K.: Remarks on Cheon's Algorithms for PairingRelated Problems. In: Takagi, T., Okamoto, T., Okamoto, E., Okamoto, T. (eds.) Pairing 2007. LNCS, vol. 4575, pp. 302-316. Springer, Heidelberg (2007)

13. Montgomery, P.: Speeding the Pollard and Elliptic Curve Methods of Factorization. Math. Comp. 48(177), 243-264 (1987)

14. Oliveira, L., López, J., Dahab, R.: TinyTate: Identity-Based Encryption for Sensor Networks, IACR Cryptology ePrint Archive, Report 2007/020 (2007)

15. Pollard, J.: Monte Carlo Methods for Index Computation (mod p). Math. Comp. 32, 918-924 (1978)

16. Shanks, D.: Class Number, a Theory of Factorization, and Genera. In: Proc. of Symp. Math. Soc., vol. 20, pp. 41-440 (1971) 